\title{
Superposability of the equations of magneto-hydrodynamics.
}

\author{
By G. Teeka RAO \\ (Received Nov. 6, 1958) \\ (Revised June 1, 1959)
}

$\S$ 0. The equations of magneto-hydrodynamics in an incompressible viscous electrically conducting fluid are given by

$$
\begin{aligned}
& \frac{\epsilon \mu}{C^{2}} \frac{\partial^{2} \vec{H}}{\partial t^{2}}-\Delta_{2} \vec{H}+\frac{4 \pi \sigma \mu}{C^{2}}\left[\frac{\partial \vec{H}}{\partial t}+\operatorname{Curl}(\vec{H} \times \vec{q})\right]=0, \\
& \frac{\partial \vec{q}}{\partial t}-\vec{q} \times \operatorname{Curl} \vec{q}+\frac{\mu}{4 \pi \rho} \vec{H} \times \operatorname{Curl} \vec{H} \\
& \quad+\operatorname{grad}\left(\frac{1}{2} \vec{q}^{2}+\frac{p}{\rho}-U\right)-r \Delta_{2} \vec{q}=0,
\end{aligned}
$$

$$
\operatorname{div} \vec{q}=0, \quad \operatorname{div} \vec{H}=0,
$$

the notations being classical. As in the case of equations of motion in hydrodynamics, here also, the equations contain non-linear terms and the solutions cannot be superposed in general. We examine below the necessary and sufficient conditions under which two solutions of the system of equations (0.1), (0.2), (0.3) can be superposed on one another, i. e., we seek the conditions such that when $\left(\vec{q}_{1}, \vec{H}_{1}, p_{1}, U_{1}\right)$ and $\left(\vec{q}_{2}, \vec{H}_{2}, p_{2}, U_{2}\right)$ are solutions of the system, $\left(\vec{q}_{1}+\vec{q}_{2}, \vec{H}_{1}+\dot{H}_{2}, p_{1}+p_{2}+\pi, U_{1}+U_{2}\right)$ is also a solution of the system. Similar investigations for the hydrodynamical case have been made in some detail [1].

$\S 1$. The equations for the first two fields are

$$
\begin{aligned}
& \begin{aligned}
& \frac{\epsilon \mu}{C^{2}} \frac{\partial^{2} \vec{H}_{i}}{\partial t^{2}}-\Delta_{2} \vec{H}_{i}+\frac{4 \pi \sigma \mu}{C^{2}}\left[\frac{\partial \vec{H}_{i}}{\partial t}+\operatorname{Curl}\left(\vec{H}_{i} \times \vec{q}_{i}\right)\right]=0, \\
& \frac{\partial q_{i}}{\partial t}-\vec{q}_{i} \times \operatorname{Curl} \vec{q}_{i}+\frac{\mu}{4 \pi \rho} \vec{H}_{i} \times \operatorname{Curl} \vec{H} \\
&+\operatorname{grad}\left(\frac{1}{2} \vec{q}_{i}{ }^{2}+\frac{p_{i}}{\rho}-U_{i}\right)=r \Delta_{2} \vec{q}_{i}{ }^{2}, \\
& \operatorname{div} \vec{q}_{i}=0, \quad \operatorname{div} \vec{H}_{i}=0 \quad(i=1,2)
\end{aligned}
\end{aligned}
$$

and the equations for the field resulting from the superposition are 


$$
\begin{gathered}
\frac{\epsilon \mu}{C^{2}} \frac{\partial^{2}\left(\vec{H}_{1}+\vec{H}_{2}\right)}{\partial t^{2}}-\Delta^{2}\left(\vec{H}_{1}+\vec{H}_{2}\right)+\frac{4 \pi \sigma \mu}{C^{2}}\left[\frac{\partial\left(H_{1}+H_{0}\right)}{\partial t}\right. \\
\left.+\operatorname{Curl}\left(\vec{H}_{1}+\vec{H}_{2}\right) \times\left(\vec{q}_{1}+\vec{q}_{2}\right)\right]=0 \\
\frac{\partial\left(\vec{q}_{1}+\vec{q}_{2}\right)}{\partial t}-\left(\vec{q}_{1}+\vec{q}_{2}\right) \times \operatorname{Curl}\left(\vec{q}_{1}+\vec{q}_{2}\right)+\frac{\mu}{4 \pi \rho}\left[\left(\vec{H}_{1}+\vec{H}_{2}\right)\right. \\
\left.\times \operatorname{Curl}\left(\vec{H}_{1}+\vec{H}_{2}\right)\right]+\operatorname{grad}\left[\frac{1}{2}\left(\vec{q}_{1}+\vec{q}_{2}\right)^{2}\right. \\
\left.+\frac{p_{1}+p_{2}+\pi}{\rho}-\left(U_{1}+U_{2}\right)\right]=r \Delta_{2}\left(\vec{q}_{1}+\vec{q}_{2}\right)
\end{gathered}
$$

From equations (1.1), (1.2), (1.4) and (1.5) we get

$$
\begin{gathered}
\operatorname{Curl}\left[\vec{H}_{1} \times \vec{q}_{2}+\vec{H}_{2} \times \vec{q}_{1}\right]=0, \\
\vec{q}_{1} \times \operatorname{Curl} \vec{q}_{2}+\vec{q}_{2} \times \operatorname{Curl} \vec{q}_{1}-\frac{\mu}{4 \pi \rho}\left(\vec{H}_{1} \times \operatorname{Curl} \vec{H}_{2}+\vec{H}_{2} \times \operatorname{Curl} \vec{H}_{1}\right) \\
-\operatorname{grad}\left(\vec{q}_{1} \cdot \vec{q}_{2}+\frac{\pi}{\rho}\right)=0 .
\end{gathered}
$$

The field $(\vec{q}, \vec{H}, p, U)$ is self superposable when

$$
\begin{aligned}
& \operatorname{Curl}(H \times q)=0 \\
& \operatorname{Curl}\left[\vec{q} \times \operatorname{Curl} \vec{q}-\frac{\mu}{4 \pi \rho} \vec{H} \times \operatorname{Curl} \vec{H}\right]=0
\end{aligned}
$$

We examine in some detail the case where the vectors $\vec{q}, \vec{H}$ are coplanar, when they are both two-dimensional and axially symmetric vectors.

§2. Two-dimensional motion

$$
\begin{gathered}
\vec{q}=q_{x} \vec{i}_{x}+q_{y} \vec{i}_{y}, \quad \vec{H}=H_{x} \vec{i}_{x}+H_{y} \vec{i}_{y}, \\
q_{x}=-\frac{\partial \psi}{\partial y}, \quad q_{y}=\frac{\partial \psi}{\partial x}, \\
H_{x}=-\frac{\partial \phi}{\partial y}, \quad H_{y}=\frac{\partial \phi}{\partial x},
\end{gathered}
$$

where the stream lines and the lines of force are given by $\psi=$ constant and $\phi=$ constant respectively.

From (1.6) we see that there exists a scalar function $F$ such that

$$
\vec{H}_{1} \times \vec{q}_{2}+\vec{H}_{2} \times \vec{q}_{1}=\operatorname{grad} F
$$

In terms of the functions $\psi$ and $\phi$ this condition becomes

$$
\frac{\delta\left(\phi_{1}, \psi_{2}\right)}{\delta(x, y)}+\frac{\delta\left(\phi_{2}, \psi_{1}\right)}{\delta(x, y)}=0
$$


Condition (1.7) now becomes

$$
\frac{\delta\left(\psi_{1}, \Delta_{2} \psi_{2}\right)}{\delta(x, y)}+\frac{\delta\left(\psi_{2}, \Delta_{2} \psi_{1}\right)}{\delta(x, y)}=\frac{\mu}{4 \pi \rho}\left[\frac{\delta\left(\phi_{1}, \Delta_{2} \phi_{2}\right)}{\delta(x, y)}+\frac{\delta\left(\phi_{2}, \Delta_{2} \phi_{1}\right)}{\delta(x, y)}\right] .
$$

§ 3. Conditions for superposability in the case of axial symmetry

$$
\begin{aligned}
& \vec{q}=q_{x} \vec{i}_{x}+q_{\bar{\omega}} \vec{i}_{\bar{\omega}}+q_{\omega} \vec{i}_{\omega}, \\
& \vec{H}=H_{x} \vec{i}_{x}+H_{\bar{\omega}} \vec{i}_{\bar{\omega}}+H_{\omega} \vec{i}_{\omega} .
\end{aligned}
$$

The conditions $\operatorname{div} \vec{q}=0$ and $\operatorname{div} \vec{H}=0$ are satisfied by taking

$$
\begin{array}{ll}
q_{x}=-\frac{1}{\omega} \frac{\partial \psi}{\partial \bar{\omega}}, & q_{\bar{\omega}}=-\frac{1}{\omega} \frac{\partial \psi}{\partial x}, \\
H_{x}=-\frac{1}{\bar{\omega}} \frac{\partial \phi}{\partial \bar{\omega}}, & H_{\bar{\omega}}=\frac{1}{\bar{\omega}} \frac{\partial \phi}{\partial x},
\end{array}
$$

and we may write $q_{\omega}=f(x, \bar{\omega})$, and $H_{\omega}=g(x, \bar{\omega})$.

From (2.2) we now may write

$$
\begin{aligned}
& -\frac{1}{\bar{\omega}}\left[\frac{\partial \phi_{1}}{\partial x} f_{2}-\frac{\partial \psi_{2}}{\partial x} g_{1}+\frac{\partial \phi_{2}}{\partial x} f_{1}-\frac{\partial \psi_{1}}{\partial x} g_{2}\right] d x \\
& \quad+\frac{1}{\bar{\omega}}\left[\frac{\partial \phi_{1}}{\partial \bar{\omega}} f_{2}-\frac{\partial \psi_{2}}{\partial \bar{\omega}} g_{1}+\frac{\partial \phi_{2}}{\partial \bar{\omega}} f_{1}-\frac{\partial \psi_{1}}{\partial \bar{\omega}} g_{2}\right] d \bar{\omega}=d F-\frac{\partial F}{\partial t} \delta t
\end{aligned}
$$

and

$$
\frac{\delta\left(\phi_{1}, \psi_{2}\right)}{\delta(x, \bar{\omega})}+\frac{\delta\left(\phi_{2}, \psi_{1}\right)}{\delta(x, \bar{\omega})}=0
$$

By means of cross differentiation we may write from (3.1) a single equation

$$
\begin{aligned}
\frac{\delta\left(\phi_{1}, f_{2}\right)}{\delta(x, \bar{\omega})}+\frac{\delta\left(\phi_{2}, f_{1}\right)}{\delta(x, \bar{\omega})}-\frac{1}{\bar{\omega}}\left[\frac{\partial \phi_{1}}{\partial x} f_{2}+\frac{\partial \phi_{2}}{\partial x} f_{1}\right] \\
=\frac{\delta\left(\psi_{1}, g_{2}\right)}{\delta(x, \bar{\omega})}+\frac{\delta\left(\psi_{2}, g_{1}\right)}{\delta(x, \bar{\omega})}-\frac{1}{\bar{\omega}}\left[\frac{\partial \psi_{1}}{\partial x} g_{2}+\frac{\partial \psi_{2}}{\partial x} g_{1}\right]
\end{aligned}
$$

From (1.7) we get

$$
\begin{aligned}
& \frac{1}{\bar{\omega}^{2}}\left[\frac{\partial \psi_{1}}{\partial x} E^{2} \psi_{2}+\frac{\partial \psi_{2}}{\partial x} E^{2} \psi_{1}+\frac{\partial}{\partial x}\left(\bar{\omega}^{2} f_{1} f_{2}\right)\right. \\
& \left.-\frac{\mu}{4 \pi \rho}\left(\frac{\partial \phi_{1}}{\partial x} E^{2} \phi_{2}+\frac{\partial \phi_{2}}{\partial x} E^{2} \phi_{1}+\frac{\partial}{\partial x}\left(\bar{\omega}^{2} g_{1} g_{2}\right)\right)\right] \overrightarrow{i_{x}} \\
& +\frac{1}{\bar{\omega}^{2}}\left[\left(\frac{\partial \psi_{1}}{\partial \bar{\omega}} E^{2} \psi_{2}+\frac{\partial \psi_{2}}{\partial \bar{\omega}} E^{2} \psi_{1}+\frac{\partial}{\partial \bar{\omega}}\left(\bar{\omega}^{2} f_{1} f_{2}\right)\right)\right. \\
& \left.-\frac{\mu}{4 \pi \rho}\left(\frac{\partial \phi_{1}}{\partial \bar{\omega}} E^{2} \phi_{2}+\frac{\partial \phi_{2}}{\partial \bar{\omega}} E^{2} \phi_{1}+\frac{\partial}{\partial \bar{\omega}^{2}}\left(\bar{\omega}^{2} g_{1} g_{2}\right)\right)\right] \overrightarrow{i_{\bar{\omega}}}
\end{aligned}
$$




$$
\begin{gathered}
+\frac{1}{\bar{\omega}^{2}}\left[\left(\frac{\partial \psi_{1}}{\partial \bar{\omega}} \frac{\partial}{\partial x}\left(\bar{\omega} f_{2}\right)-\frac{\partial \psi_{1}}{\partial x} \frac{\partial}{\partial \bar{\omega}}\left(\bar{\omega} f_{2}\right)+\frac{\partial \psi_{2}}{\partial \bar{\omega}} \frac{\partial}{\partial x}\left(\bar{\omega} f_{1}\right)-\frac{\partial \psi_{2}}{\partial x} \frac{\partial}{\partial \bar{\omega}}\left(\bar{\omega} f_{1}\right)\right.\right. \\
\left.-\frac{\mu}{4 \pi \rho}\left(\frac{\partial \phi_{1}}{\partial \bar{\omega}} \frac{\partial}{\partial x}\left(\bar{\omega} g_{2}\right)-\frac{\partial \phi_{1}}{\partial x} \frac{\partial}{\partial \bar{\omega}}\left(\bar{\omega} g_{2}\right)+\frac{\partial \phi_{2}}{\partial \bar{\omega}} \frac{\partial}{\partial x}\left(\bar{\omega} g_{1}\right)-\frac{\partial \phi_{2}}{\partial x} \frac{\partial}{\partial \bar{\omega}}\left(\bar{\omega} g_{1}\right)\right)\right] \vec{i}_{\omega} \\
=\operatorname{grad}\left(\vec{q}_{1} \cdot \vec{q}_{2}+\frac{\pi}{\rho}\right)
\end{gathered}
$$

where $E^{2}$ is the differential operator

$$
\frac{\partial^{2}}{\partial x^{2}}+\frac{\partial^{2}}{\partial \bar{\omega}^{2}}-\frac{1}{\bar{\omega}} \frac{\partial}{\partial \bar{\omega}}
$$

Writing $\frac{\pi}{\rho}+\vec{q}_{1} \cdot \vec{q}_{2}=G$ we have the right hand of (3.4) in the form

$$
\frac{\partial G}{\partial x} \overrightarrow{i_{x}}+\frac{\partial G}{\partial \bar{\omega}} \vec{i}_{\bar{\omega}}+\frac{1}{\bar{\omega}} \frac{\partial G}{\partial \bar{\omega}} \vec{i}_{\omega} .
$$

Since $\vec{q}_{1}$ and $\vec{q}_{2}$ are axially symmetric and $\pi$ also is to be axially symmetric, we have $\frac{\partial G}{\partial \omega}=0$. In view of this we get from (3.4)

$$
\begin{gathered}
\frac{1}{\bar{\omega}^{2}}\left[\frac{\partial \psi_{1}}{\partial x} E^{2} \psi_{2}+\frac{\partial \psi_{2}}{\partial x} E^{2} \psi_{1}+\frac{\partial}{\partial x}\left(\bar{\omega}^{2} f_{1} f_{2}\right)\right. \\
\left.-\frac{\mu}{4 \pi \rho}\left\{\frac{\partial \phi_{1}}{\partial x} E^{2} \phi_{2}+\frac{\partial \phi_{2}}{\partial x} E^{2} \phi_{1}+\frac{\partial}{\partial x}\left(\bar{\omega}^{2} g_{1} g_{2}\right)\right\}\right] d x \\
+\frac{1}{\bar{\omega}^{2}}\left[\frac{\partial \psi_{1}}{\partial \bar{\omega}} E^{2} \psi_{2}+\frac{\partial \psi_{2}}{\partial \bar{\omega}} E^{2} \psi_{1}+\frac{\partial}{\partial \bar{\omega}}\left(\bar{\omega}^{2} f_{1} f_{2}\right)\right. \\
\left.\quad-\frac{\mu}{4 \pi \rho}\left\{\frac{\partial \phi_{1}}{\partial \bar{\omega}} E^{2} \phi_{2}+\frac{\partial \phi_{2}}{\partial \bar{\omega}} E^{2} \phi_{1}+\frac{\partial}{\partial \bar{\omega}}\left(\bar{\omega}^{2} g_{1} g_{2}\right)\right\}\right] d \bar{\omega} \\
=d G-\frac{\partial G}{\partial t} \delta t
\end{gathered}
$$

and

$$
\frac{\delta\left(\psi_{1}, \bar{\omega} f_{2}\right)}{\delta(x, \bar{\omega})}+\frac{\delta\left(\psi_{2}, \bar{\omega} f_{1}\right)}{\delta(x, \bar{\omega})}=\frac{\mu}{4 \pi \rho}\left\{\frac{\delta\left(\phi_{1}, \bar{\omega} g_{2}\right)}{\delta(x, \bar{\omega})} \frac{\delta\left(\phi_{2}, \bar{\omega} g_{1}\right)}{\delta(x, \bar{\omega})}\right\}
$$

From (3.6) we may derive by means of cross differentiation

$$
\begin{gathered}
\\
\frac{\delta\left(\psi_{1}, E^{2} \psi_{2}\right)}{\delta(x, \bar{\omega})}+\frac{\delta\left(\psi_{2}, E^{2} \psi_{1}\right)}{\delta(x, \bar{\omega})}-\frac{2}{\bar{\omega}}\left\{\frac{\partial \psi_{1}}{\partial x} E^{2} \psi_{2}+\frac{\partial \psi_{2}}{\partial x} E^{2} \psi_{1}+\frac{\partial}{\partial x}\left(\bar{\omega}^{2} f_{1} f_{2}\right)\right\} \\
=\frac{\mu}{4 \pi \rho}\left[\frac{\delta\left(\phi_{1}, E^{2} \phi_{2}\right)}{\delta(x, \bar{\omega})}+\frac{\delta\left(\phi_{\cdots}, E^{2} \phi_{1}\right)}{\delta(x, \bar{\omega})}-\frac{2}{\bar{\omega}}\left\{\frac{\partial \phi_{1}}{\partial x} E^{2} \phi_{2}+\frac{\partial \phi_{2}}{\partial x} E^{2} \phi_{1}+\frac{\partial}{\partial x}\left(\bar{\omega}^{2} g_{1} g_{2}\right)\right\}\right] .
\end{gathered}
$$

Equation (3.6) can also be written in the form

$$
d\left(\begin{array}{c}
\pi \\
\rho
\end{array}\right)=\frac{1}{\bar{\omega}^{2}}\left[d \psi_{1} E^{2} \psi_{2}+d \psi_{2} E^{2} \psi_{1}+d\left(\bar{\omega}^{2} f_{1} f_{2}\right)-\frac{\mu}{4 \pi \rho}\left\{\left(d \phi_{1} E^{2} \phi_{2}\right.\right.\right.
$$




$$
\left.\left.+d \phi_{2} E^{2} \phi_{1}+d\left(\bar{\omega}^{2} g_{1} g_{2}\right)\right\}\right]-d\left\{\frac{1}{\omega^{2}}\left(\frac{\partial \psi_{1}}{\partial x} \frac{\partial \psi_{2}}{\partial x}+\frac{\partial \psi_{1}}{\partial \omega} \frac{\partial \psi_{2}}{\partial \omega}\right)\right\}
$$

and determines the adjusted pressure.

We now indicate two specific examples of flows that are superposable.

In the case where the fields $\vec{q}$ and $\vec{H}$ are both coplanar and independent of the space coordinate $Z$, we can assume them in the form given in (2.1), or using polar coordinates $r, \theta$ in the $x o y$-plane, we can also take them as

$$
\begin{aligned}
\vec{q}_{i} & =\left(\frac{1}{r} \frac{\partial \psi_{i}}{\partial \theta},-\frac{\partial \psi_{i}}{\partial r}, o\right), \\
\vec{H}_{i} & =\left(\frac{1}{r} \frac{\partial \phi_{i}}{\partial \theta},-\frac{\partial \phi_{i}}{\partial r}, o\right) \quad(i=1,2) .
\end{aligned}
$$

The necessary and sufficient conditions (viz., 2.3, 2.4) for the addition of two can be written in the form

$$
\begin{aligned}
{\left[-\frac{\partial}{\partial \theta}\left\{\frac{1}{r^{2}}\left(\frac{\partial\left(\psi_{1}, \phi_{2}\right)}{\partial(r, \theta)}+\frac{\partial\left(\psi_{2}, \phi_{1}\right)}{\partial(r, \theta)}\right)\right\},\right.} \\
\left.\quad \frac{\partial}{\partial r}\left\{\frac{1}{r}\left(\frac{\partial\left(\psi_{1}, \phi_{2}\right)}{\partial(r, \theta)}+\frac{\partial\left(\psi_{2}, \phi_{1}\right)}{\partial(r, \theta)}\right)\right\}, o\right]=0
\end{aligned}
$$

and

$$
\begin{aligned}
\frac{1}{r}[o, o, & \left.+\frac{\partial\left(\psi_{1}, \Delta_{2} \psi_{2}\right)}{\partial(r, \theta)}+\frac{\partial\left(\psi_{2}, \Delta_{2} \psi_{1}\right)}{\partial(r, \theta)}\right] \\
& -\left[o, o, \frac{\mu}{4 \pi \rho} \frac{1}{r}\left(\frac{\partial\left(\phi_{1}, \Delta_{2} \phi_{2}\right)}{\partial(r, \theta)}+\frac{\partial\left(\phi_{2}, \Delta_{2} \phi_{1}\right)}{\partial(r, \theta)}\right)\right]=0 .
\end{aligned}
$$

Writing $\sqrt{\frac{\mu}{4 \pi \rho}} \phi_{i}=\bar{\phi}_{i}, i=1,2$, these equations give

$$
\begin{gathered}
\frac{\partial}{\partial \theta}\left[\frac{\partial\left(\psi_{1}, \bar{\phi}_{2}\right)}{\partial(r, \theta)}+\frac{\partial\left(\psi_{2}, \overline{\phi_{1}}\right)}{\partial(r, \theta)}\right]=0, \\
\frac{\partial}{\partial r}\left[\frac{1}{r} \frac{\partial\left(\psi_{1}, \overline{\phi_{2}}\right)}{\partial(r, \theta)}+\frac{\partial\left(\psi_{2}, \overline{\phi_{1}}\right)}{\partial(r, \theta)}\right]=0, \\
\frac{\partial\left(\psi_{1}, \Delta_{2} \psi_{2}\right)}{\partial(r, \theta)}+\frac{\partial\left(\psi_{2}, \Delta_{2} \psi_{1}\right)}{\partial(r, \theta)}-\frac{\partial\left(\overline{\phi_{1}}, \Delta_{2}, \overline{\phi_{2}}\right)}{\partial(r, \theta)}-\frac{\partial\left(\overline{\phi_{2}}, \Delta_{2}, \overline{\phi_{1}}\right)}{\partial(r, \theta)}=0 .
\end{gathered}
$$

Cataldo Agostinelli* [2] has shown that the fields (4.1) and (4.2) satisfy the fundamental equations $(0.1)$ and $(0.2)$ if

$$
\begin{aligned}
& \phi_{i}=-\frac{1}{2} h_{i} r^{2}+f_{i}(r, \theta, t), \\
& \psi_{i}=-\frac{1}{2} \omega_{i} r^{2}+\beta f_{i}(r, \theta, t) \quad(i=1,2),
\end{aligned}
$$

* The results of [2] referred to here were available to the author only from the Math. Rev., 18 (1957), p. 849. 
where $h_{i}, \omega_{i}, \beta$ are constants and

$$
\begin{aligned}
& \text { (i) } \Delta_{2} f_{i}=0 \text { or (ii) } \beta=\alpha=\sqrt{\frac{\mu}{4 \pi \rho}} \text { and } \\
& \qquad \Delta_{2}\left(\frac{\partial f_{i}}{\partial t}-\gamma \Delta_{2} f_{i}+\left(\omega_{i}-\alpha h_{i}\right) \frac{\partial f_{i}}{\partial \theta}\right)=0 .
\end{aligned}
$$

It is easily seen that fields given in (4.8) and (4.9) satisfy the condition (4.7) and from (4.5) and (4.6) we get

$$
\begin{gathered}
\frac{\partial\left(\psi_{1}, \bar{\phi}_{2}\right)}{\partial(r, \theta)}+\frac{\partial\left(\psi_{2}, \bar{\phi}_{1}\right)}{\partial(r, \theta)}=g(r), \\
\frac{\partial}{\partial r}\left[\frac{g(r)}{r}\right]=0 .
\end{gathered}
$$

Whence we deduce that

$$
f_{K}(r)=g_{K}(r)+a_{K} \theta+b_{K} \quad(K=1,2)
$$

with $g_{K}(r)=$ a harmonic function.

We may therefore write

$$
\begin{aligned}
& \phi_{i}=-\frac{1}{2} h_{i} r^{2}+\left(A_{i}+B_{i} \log r\right)+a_{i} \theta+b_{i} \\
& \psi_{i}=-\frac{1}{2} \omega_{i} r^{2}+\beta\left(A_{i}+B_{i} \log r+a_{i} \theta+b_{i}\right) \quad(i=1,2)
\end{aligned}
$$

and the corresponding fields are

$$
\begin{aligned}
\vec{q}_{i} & =\left(\frac{a_{i}}{r} \beta, \omega_{i} r-\beta \frac{B_{i}}{r}, o\right), \\
\vec{H}_{i} & =\left(\frac{a_{i}}{r}, h_{i} r-\frac{B_{i}}{r}, o\right) .
\end{aligned}
$$

Hence we have the result that

The fields defined in (4.15), (4.16) are superposable. When the functions $f_{i}(r, \theta)$ of (4.8), (4.9)] satisfy the condition

$$
\Delta_{2}\left(-\gamma \Delta_{2} f_{i}+\left[\omega_{i}-\alpha h_{i}\right] \frac{\partial f_{i}}{\partial \theta}\right)=0 \quad(i=1,2) .
$$

The conditions (4.5) and (4.6) and (4.7) are reduced to the form

$$
\begin{gathered}
\frac{\partial\left(\psi_{1}, \phi_{2}\right)}{\partial(r, \theta)}+\frac{\partial\left(\psi_{2}, \phi_{1}\right)}{\partial(r, \theta)}=g(r), \\
\frac{\partial}{\partial r}\left[\frac{g(r)}{r}\right]=0
\end{gathered}
$$

and

$$
\left(\omega_{1}-\alpha h_{1}\right) \frac{\partial}{\partial \theta}\left(\Delta_{2} f_{2}\right)+\left(\omega_{2}-\alpha h_{2}\right) \frac{\partial}{\partial \theta}\left(\Delta_{2} f_{1}\right)=0
$$


We may write (4.19) also in the form (compare (4.17))

$$
\Delta_{2}\left(-r \Delta_{2} f_{2}\right)+\Delta_{2}\left(-r \Delta_{2} f_{1}\right)=0 \text {. }
$$

After some calculation we get from this that

$$
f_{K}=g_{K}(r)+a_{K} \theta+b_{K} \quad(K=1,2),
$$

where $g_{K}(r)$ is a biharmonic function, i. e.,

$$
g_{K}(r)=C_{K}+d_{K} \log r+\frac{A_{K}}{4} r^{2}+\frac{B_{K}}{4}(\log r-1) r^{2} .
$$

Hence we have

$$
\begin{aligned}
\psi_{i}= & -\frac{1}{2} \omega_{i} r^{2}+\left[C_{i}+D_{i} \log r+\frac{A_{i}}{4} r^{2}+\frac{B_{i}}{4}(\log r-1) r^{2}\right] \beta \\
& +\beta\left(a_{K} \theta+b_{K}\right), \\
\phi_{i}= & -\frac{1}{2} h_{i} r^{2}+\left[C_{i}+D_{i} \log r+\frac{A_{i}}{4} r^{2}+\frac{B_{i}}{4}(\log r-1) r^{2}\right] \\
& +a_{K} \theta+b_{K}
\end{aligned}
$$

and the corresponding fields are

$$
\begin{aligned}
\vec{q}_{i} & =\left[\frac{\beta a_{i}}{r}, \omega_{i} r-\left(\frac{D_{i}}{r}+\frac{A_{i}}{2} r+\frac{B_{i}}{2} r(\log r-1)+\frac{B_{i}}{4} r\right) \beta+o, o\right], \\
\vec{H}_{i} & =\left[\frac{a_{i}}{r},\left(h_{i} r-\frac{D_{i}}{r}-A_{i} r-B_{i} r(\log r-1)-\frac{B_{i}}{4} r, 0\right)\right] .
\end{aligned}
$$

We have therefore the result:

The motions given by (4.24) and (4.25) are superposable.

The author wishes to thank the referee for his valuable suggestions for improving the first version of my paper.

Department of Mathematics

Arts and Science College Warangal, India.

\section{References}

[1] R. Ballabh, Two dimensional superposable motions, J. of Indian Math. Soc., (1952), 16, 191-197.

[2] C. Agostinelli, The solutions of the equations of magneto-hydrodynamics in cylindrical coordinates, Math. Rev., 18 (1957), p. 849. 